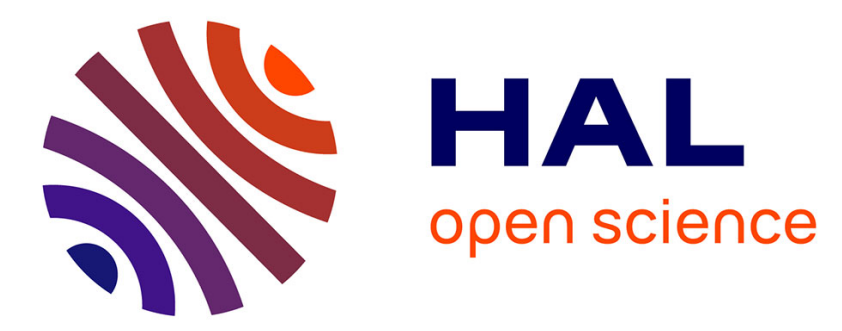

\title{
Dynamic Control of Parallel Robots Driven by Flexible Cables and Actuated by Position-Controlled Winches
}

Jeremy Begey, Loic Cuvillon, Maximilian Lesellier, Marc Gouttefarde, Jacques Gangloff

\section{- To cite this version:}

Jeremy Begey, Loic Cuvillon, Maximilian Lesellier, Marc Gouttefarde, Jacques Gangloff. Dynamic Control of Parallel Robots Driven by Flexible Cables and Actuated by Position-Controlled Winches. IEEE Transactions on Robotics, 2019, 35 (1), pp.286-293. 10.1109/TRO.2018.2875415 . lirmm02087410v2

\section{HAL Id: lirmm-02087410 \\ https://hal-lirmm.ccsd.cnrs.fr/lirmm-02087410v2}

Submitted on 12 Jan 2021

HAL is a multi-disciplinary open access archive for the deposit and dissemination of scientific research documents, whether they are published or not. The documents may come from teaching and research institutions in France or abroad, or from public or private research centers.
L'archive ouverte pluridisciplinaire HAL, est destinée au dépôt et à la diffusion de documents scientifiques de niveau recherche, publiés ou non, émanant des établissements d'enseignement et de recherche français ou étrangers, des laboratoires publics ou privés. 


\section{Dynamic Control of Parallel Robots Driven by Flexible Cables and Actuated by Position-Controlled Winches}

\author{
Jeremy Begey, Loïc Cuvillon, Maximilien Lesellier, \\ Marc Gouttefarde and Jacques Gangloff
}

\begin{abstract}
An alternative approach to standard computed torque with feedback linearization is proposed in this work to control cable-driven parallel robots (CDPRs) with highly flexible cables. Exteroceptive feedback is used to measure the end-effector Cartesian position at a high sampling rate. Stability is demonstrated using singular perturbation theory. The proposed control scheme is experimentally validated on a planar 3-degree-of-freedom CDPR and its efficiency is assessed by comparison to a simple kinematic control law.
\end{abstract}

Index Terms-Parallel robots, Dynamics,Flexible cables, Singular perturbation, Motion control.

\section{INTRODUCTION}

The main advantages of Cable-Driven Parallel Robots (CDPRs) include a large workspace, low inertia links, cost-effective scalability and potentially heavy payload capabilities. Their main drawbacks are high flexibility and relatively low positioning accuracy. Advanced dynamic control with exteroceptive feedback can compensate for these drawbacks by improving accuracy and rejecting the effects of flexibility. Some CDPRs use very flexible cables (very long cables or cables using a flexible material like polymer). This paper shows that flexibility can be an advantage from the control point of view since they yield lower frequency oscillations that can be more easily handled by position-controlled actuators.

Dynamic control of a Parallel Robot (PR) in the Cartesian space coordinates is more suitable than in the joint space coordinates as highlighted by Paccot et al. in [1]. Computational efficiency, improved task robustness and better accuracy can be expected when using exteroceptive measurements of the end-effector pose. If the exteroceptive sensor is a camera and the task is defined in image space (2D) or Cartesian space (3D), the control law can be designed using the visual servoing framework [2], [3], [4].

All control strategies reviewed in [1] involve partial or complete inversion of the robot nonlinear dynamics. According to Cheng et al. [5], three control strategies may be implemented: Simple PD control in Cartesian space, augmented PD control and computed-torque control. Computed-torque control, also called inverse dynamics control or, in the most general context, feedback linearization, effectively achieves a decoupling and linearization of the manipulator dynamics.

Many of these control strategies suited for rigid-link PRs have been adapted to CDPRs. Compared to rigid-link PRs, a distinctive feature of CDPRs is the unidirectional nature of the forces applied by the cables on the end-effector [6]. Consequently, a tension distribution algorithm must be implemented to ensure positiveness of cable tension in case of actuation redundancy [7], [8], [9].

Several CDPR control studies are based on the assumption that cables are rigid. Some of them are controlling the CDPR in the joint space [10], [11]. Others are controlling the robot in the Cartesian space [6], [12], [13], [14]. When controlling the CDPR in Cartesian space, pose feedback can be provided by forward kinematics [6], [12] or visual feedback [13], [14]. In all these works, the control input is a torque reference signal sent to current regulation of the winch actuator drive.

Other CDPR control studies are based on the assumption that is made in this work, i.e. that cables are elastic. None of them use

J. Begey, L. Cuvillon and J. Gangloff are members of the ICube laboratory, University of Strasbourg, France. M. Lesellier and M. Gouttefarde are members of LIRMM, Université de Montpellier, CNRS, France. visual feedback to measure the pose of the end-effector. Instead, forward kinematics is used to estimate this pose. An analysis of CDPR vibration modes is given by Diao et al. in [15]. It shows that transversal cable flexibility can be neglected with respect to axial flexibility. Accordingly, in most of the papers cited below, the cables are modeled as straight line segments with an axial stiffness.

A cascade control architecture is proposed by Meunier et al. in [16] to control the end-effector pose of a CDPR with long elastic cables. The higher level controller (outer loop) regulates the distance between the winch output and the end-effector anchor point to obtain the desired pose. Its control signal is a vector of cable tension references. To ensure fast convergence of elastic cable tensions toward the references, an inner loop controls unstretched cable lengths by sending control signals to a winch angular position loop. In this work, the outer loop is a PID controller and the inverse dynamic model (IMD) of the end-effector is used to compute the desired cable tensions. This cascade controller is evaluated in simulation but no proof of stability is given for this architecture. Moreover, the dynamics of the winch angular position loop are neglected in the design of the inner loop.

A more complex lumped-mass cable model is used by Caverly et al. in [17], [18]. A model of the CDPR dynamics is derived taking into account the cables, the end-effector and the inertia of the torquecontrolled winches. A passivity-based control is proposed to ensure stable tracking of the desired CDPR tip position.

Khosravi and Jamshidifar explicitly addressed the rejection in Cartesian space of the vibrations induced by the axial flexibility of massless cables in [19] and [20]. Similarly to the controller proposed by Ghorbel and Spong [21] for serial manipulators with flexible joints, a composite control involving a computed-torque controller and a constant feedback on the cable stretching velocity is studied in [19]. No assessment is provided on a physical setup and no detail is given on how to measure or estimate the end-effector pose and cable stretching required for control. A detailed proof of stability based on singular perturbation theory [22], [23] is provided, where high cable stiffness is assumed such that vibrations can be considered as fast dynamics. This proof extends the partial proof of stability given in [21] for serial robots, where it is assumed that the joint stiffness is large compared to other system parameters.

To the best of the authors' knowledge, [20] is the most recent work on CDPRs dealing with vibration rejection control. Based on a first order linearization of the CDPR dynamics along a desired trajectory, a decoupled model of the vibration dynamics is extracted. This model is time variant due to the non-constant stiffness of the CDPR end-effector within its workspace. Thus, a linear parametervarying controller (LPV) is designed to guarantee the stability of the vibration rejection across the CDPR workspace. This approach was tested experimentally. Acceleration and velocity measurements of the end-effector needed for the LPV controller are provided by an embedded 6-axis IMU. A similar approach for vibration rejection has been proposed by Weber in [24]. Instead of an LPV controller designed in Cartesian space, the controller is designed after mapping the motion into the modal space in order to decouple the vibration modes.

When controlling the actuator torque directly, as in the control of serial robots with flexible joints through a state feedback linearization [25] and as in most of the previously cited works on CDPR, the bandwidths of the torque and of the motor current loop are the same, i.e. usually more than $1 \mathrm{kHz}$. In this case, it is completely justified to consider the actuator as a perfect torque generator. However, if the motor is associated with a gearbox as it is often the case except for rare direct-drive setups, frictions and especially dry frictions may add significant nonlinearities to the model. Cascade control may be 
a solution to reject these nonlinearities with a fast inner loop like in [16]. The present work shares the same root idea as Meunier et al. [16] that high cable flexibility may be an opportunity from a control point of view. In this case, the vibration modes of the CDPR have frequencies much lower than the bandwidth of low-level winch angular position control loops. These control loops have a linearizing effect on nonlinearities like dry friction.

The same idea is behind the inner velocity or position loops proposed among other cascade controllers in [26], [27] for the control of series elastic actuators (SEA) [28]. The system composed of the winch motor, its gearbox and the elastic cable can indeed be seen as a SEA. One benefit of SEA, that also applies to CDPR with a low cable stiffness, is the transformation of a force sensing problem into a position sensing problem. Indeed, the force can be measured accurately by the elongation of the elastic component, i.e. the cable in the case of CDPRs.

This paper is based on the novel idea that, for CDPRs with flexible cables, the winch angular position can be considered as the fast variable whereas the end-effector Cartesian pose is the slow variable in a singular perturbation framework. The main contribution of the paper is to prove the stability of the whole system controlled at the outer level with a classical feedback linearization scheme. This approach is validated experimentally on a planar 3-cable suspended CDPR. Feedback linearization needs pose and velocity feedback which are provided by a $500 \mathrm{~Hz}$ eye-to-hand camera and an embedded IMU.

This paper is organized as follows. Section II presents the modeling of CDPR dynamics with elastic cables. The control scheme is explained in section III. Proof of stability is derived in section IV. Experimental setup and results are presented in section V.

\section{MODELING}

\section{A. Cable Modeling}

The CDPR might be suspended or fully constrained.

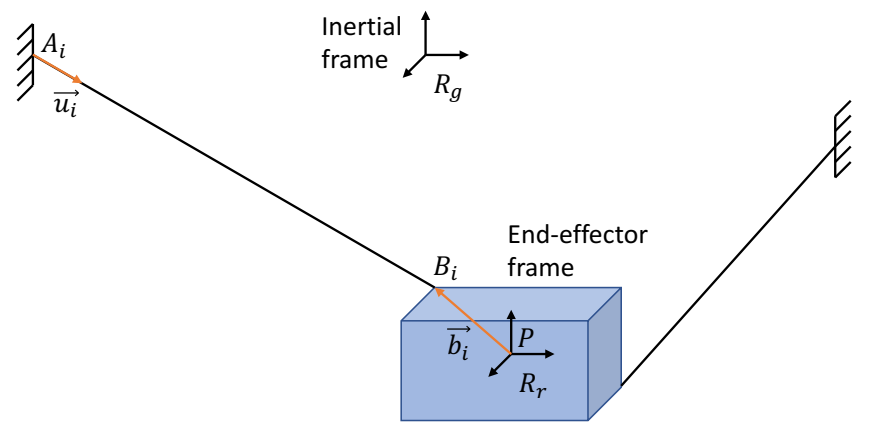

Fig. 1. CDPR general definitions.

As shown in Fig. 1, the vector $\vec{u}_{i}$ is a unit vector giving the direction of the $i^{\text {th }}$ cable. Points $A_{i}$ and $B_{i}$ are its attachment points to the base frame and to the end-effector, respectively.

The approach proposed in this paper is suited for CDPRs whose cable sagging can be neglected. When sagging is negligible, the cable can be effectively modeled by a linear spring. Studies on cable characteristics show that cables usually have a nonlinear relationship between their tension and elongation [29]. In the sequel, it is assumed that this relationship can be linearized, and thus that, within nominal tension bounds [30], the linear spring model is accurate.

Accordingly, the cable is modeled as a linear spring with a specific stiffness $k_{s}=e s(\mathrm{~N})$ related to its Young modulus $e\left(\mathrm{~N} \mathrm{~m}^{-2}\right)$ and its cross-section $s\left(\mathrm{~m}^{2}\right)$. The axial stiffness of the cable is given by:

$$
k=\frac{k_{s}}{l_{2}}=\frac{e s}{l_{2}}
$$

In (1), $l_{2}$ denotes the free length of the cable, i.e. its length when its tension is equal to zero. Winding the cable around its winch reduces $l_{2}$, and therefore increases the cable axial stiffness. Vibrations occurring at the CDPR end-effector are mainly due to the axial stiffness of the cables [15].

\section{B. Dynamics}

1) End-effector: Newton-Euler equations (2) describe the combined translational and rotational dynamics of a CDPR end-effector written in an inertial frame $R_{g}$ :

$$
\left[\begin{array}{cc}
m \mathbf{I}_{3 \times 3} & \mathbf{0}_{3 \times 3} \\
\mathbf{0}_{3 \times 3} & \mathbf{I}_{\mathbf{g}}
\end{array}\right]\left[\begin{array}{c}
\ddot{\mathbf{p}} \\
\dot{\boldsymbol{\omega}}
\end{array}\right]+\left[\begin{array}{c}
\mathbf{0}_{3 \times 1} \\
\boldsymbol{\omega} \times \mathbf{I}_{\mathbf{g}} \boldsymbol{\omega}
\end{array}\right]=\left[\begin{array}{c}
\mathbf{F} \\
\mathbf{N}
\end{array}\right]+\left[\begin{array}{c}
m \mathbf{g} \\
\mathbf{0}_{3 \times 1}
\end{array}\right]
$$

where $m$ is the end-effector mass, $\mathbf{g}$ is the gravity acceleration vector, $\ddot{\mathbf{p}}$ is the acceleration of the end-effector center of gravity $P$, vector $\dot{\omega}$ is the time derivative of the end-effector angular velocity, $\mathbf{F}$ and $\mathbf{N}$ are the force and moment vectors applied by the cables to the end-effector at point $P$. Matrix $\mathbf{I}_{\mathbf{g}}$ is the end-effector inertia tensor. The vectors and the inertia tensor are all expressed in the robot base frame $R_{g}$ (see Fig. 1).

Let $\mathbf{R}_{\mathbf{g r}}$ be the rotation matrix from the inertial frame $\left(R_{g}\right)$ to the robot end-effector frame $\left(R_{r}\right)$. Thus, the end-effector inertia tensor expressed in the inertial frame $R_{g}$ is given by: $\mathbf{I}_{\mathbf{g}}=\mathbf{R}_{\mathrm{gr}} \mathbf{I}_{\mathbf{r}} \mathbf{R}_{\mathrm{gr}}^{\mathbf{T}}$ with $\mathbf{I}_{\mathbf{r}}$ the constant inertia tensor of the end-effector expressed in $R_{r}$.

Let $\mathbf{J}$ be the inverse Jacobian of the robot mapping the velocity twist of the end-effector to the joint velocities $\mathbf{i}_{\mathbf{1}}=\left[\cdots \dot{l}_{1_{i}} \cdots\right]^{T}$, $i_{1_{i}}$ being the time derivative of the distance $A_{i} B_{i}$ between the cable end points:

$$
\mathrm{i}_{1}=\mathbf{J}\left[\begin{array}{c}
\dot{\mathbf{p}} \\
\omega
\end{array}\right]
$$

Furthermore, $\mathbf{J}^{T}$ maps the cable tensions $\boldsymbol{\tau}$ to the wrench $\mathbf{f}=$ $\left[\mathbf{F}^{T} \mathbf{N}^{T}\right]^{T}$ applied by the cables to the end-effector:

$$
\mathbf{f}=-\mathbf{J}^{T} \boldsymbol{\tau}
$$

The inverse Jacobian transpose of a CDPR is given by:

$$
\mathbf{J}^{T}=\left[\begin{array}{ccc}
\cdots & { }^{g} \mathbf{u}_{\mathbf{i}} & \cdots \\
\cdots & { }^{g} \mathbf{b}_{\mathbf{i}} \times{ }^{g} \mathbf{u}_{\mathbf{i}} & \cdots
\end{array}\right]
$$

with $\mathbf{u}_{\mathbf{i}}$ the unit vector giving the direction of the $i^{\text {th }}$ cable from its end point on the base frame to its end point on the end-effector and $\mathbf{b}_{\mathbf{i}}=\overrightarrow{P B_{i}}$ the vector from the end-effector center of gravity $P$ to the end point $B_{i}$ (see Fig. 1).

Using equation (4), equation (2) becomes:

$$
\left[\begin{array}{cc}
m \mathbf{I}_{3 \times 3} & \mathbf{0}_{3 \times 3} \\
\mathbf{0}_{3 \times 3} & \mathbf{I}_{\mathbf{g}}
\end{array}\right]\left[\begin{array}{c}
\ddot{\mathbf{p}} \\
\dot{\boldsymbol{\omega}}
\end{array}\right]+\left[\begin{array}{c}
\mathbf{0}_{3 \times 1} \\
\boldsymbol{\omega} \times \mathbf{I}_{\mathbf{g}} \boldsymbol{\omega}
\end{array}\right]=-\mathbf{J}^{T} \boldsymbol{\tau}+\left[\begin{array}{c}
m \mathbf{g} \\
\mathbf{0}_{3 \times 1}
\end{array}\right]
$$

Let $\mathbf{X}=\left[\begin{array}{ll}\mathbf{p}^{T} & \boldsymbol{\phi}^{T}\end{array}\right]^{T}$ be the vector describing the pose of the CDPR end-effector with respect to the inertial frame $R_{g}$. Vector $\phi$ contains angular coordinates (e.g. Euler angles) describing the rotation $\mathbf{R}_{\mathrm{gr}}(\phi)$. It is well known that the time derivative of a pose vector is related to the corresponding twist by a Jacobian matrix. Let us call $\mathbf{S}^{\prime}$ this $6 \times 6$ matrix such that $\left[\dot{\mathbf{p}}^{T} \boldsymbol{\omega}^{T}\right]^{T}=\mathbf{S}^{\prime} \dot{\mathbf{X}}$ where:

$$
\mathbf{S}^{\prime}=\left[\begin{array}{cc}
\mathbf{I}_{3 \times 3} & \mathbf{0} \\
\mathbf{0} & \mathbf{S}(\boldsymbol{\phi})
\end{array}\right]
$$

and $\mathbf{S}(\phi)$ denotes the matrix mapping the time derivative of $\phi$ to the angular velocity vector $\omega$ :

$$
\omega=\mathbf{S}(\phi) \dot{\phi}
$$

$\mathbf{S}$ is a $3 \times 3$ identity matrix when $\phi$ is zero, so that it is sometimes neglected in the literature since CDPRs are usually not performing large angular displacements. Here, for the sake of generality, this 
term is not neglected. Using (3), let us define $\tilde{\mathbf{J}}$ which maps the time derivative $\dot{\mathbf{X}}$ of the pose to joint velocities $\dot{\mathrm{i}}_{1}$ :

$$
\mathrm{i}_{1}=\tilde{\mathbf{J}} \dot{\mathbf{X}}
$$

with:

$$
\tilde{\mathbf{J}}=\mathbf{J S}^{\prime}
$$

Using (8) in (6) yields:

$$
\begin{aligned}
& {\left[\begin{array}{cc}
m \mathbf{I}_{3 \times 3} & \mathbf{0}_{3 \times 3} \\
\mathbf{0}_{3 \times 3} & \mathbf{I}_{\mathbf{g}} \mathbf{S}
\end{array}\right]\left[\begin{array}{c}
\ddot{\mathbf{p}} \\
\ddot{\boldsymbol{\phi}}
\end{array}\right]+\left[\begin{array}{c}
\mathbf{0}_{3 \times 1} \\
\mathbf{I}_{\mathbf{g}} \dot{\mathbf{S}} \dot{\boldsymbol{\phi}}+\mathbf{S} \dot{\boldsymbol{\phi}} \times \mathbf{I}_{\mathbf{g}} \mathbf{S} \dot{\boldsymbol{\phi}}
\end{array}\right]=} \\
& -\mathbf{J}^{T} \boldsymbol{\tau}+\left[\begin{array}{c}
m \mathbf{g} \\
\mathbf{0}_{3 \times 1}
\end{array}\right]
\end{aligned}
$$

Noting that $\tilde{\mathbf{J}}^{T}=\mathbf{S}^{\prime T} \mathbf{J}^{T}$, equation (11) can be rewritten using $\tilde{\mathbf{J}}$ which gives:

$$
\begin{array}{r}
{\left[\begin{array}{cc}
m \mathbf{I}_{3 \times 3} & \mathbf{0}_{3 \times 3} \\
\mathbf{0}_{3 \times 3} & \mathbf{S}^{T} \mathbf{I}_{\mathbf{g}} \mathbf{S}
\end{array}\right]\left[\begin{array}{c}
\ddot{\mathbf{p}} \\
\ddot{\boldsymbol{\phi}}
\end{array}\right]+\left[\begin{array}{c}
\mathbf{0}_{3 \times 1} \\
\mathbf{S}^{T}\left(\mathbf{I}_{\mathbf{g}} \dot{\mathbf{S}} \dot{\boldsymbol{\phi}}+\mathbf{S} \dot{\boldsymbol{\phi}} \times \mathbf{I}_{\mathbf{g}} \mathbf{S} \dot{\boldsymbol{\phi}}\right)
\end{array}\right]=} \\
-\tilde{\mathbf{J}}^{T} \boldsymbol{\tau}+\left[\begin{array}{c}
m \mathbf{g} \\
\mathbf{0}_{3 \times 1}
\end{array}\right]
\end{array}
$$

Introducing $\mathbf{M}, \mathbf{C}$ and $\mathbf{G}=\left[\begin{array}{c}-m \mathbf{g} \\ \mathbf{0}_{3 \times 1}\end{array}\right]$, equation (12) can be written in the classical form:

$$
\mathbf{M}(\mathbf{X}) \ddot{\mathbf{X}}+\mathbf{C}(\dot{\mathbf{X}}, \mathbf{X}) \dot{\mathbf{X}}+\mathbf{G}=-\tilde{\mathbf{J}}^{T} \boldsymbol{\tau}
$$

with

$$
\mathbf{M}(\mathbf{X})=\left[\begin{array}{cc}
m \mathbf{I}_{3 \times 3} & \mathbf{0}_{3 \times 3} \\
\mathbf{0}_{3 \times 3} & \mathbf{S}^{T} \mathbf{I}_{\mathbf{g}} \mathbf{S}
\end{array}\right]
$$

and

$$
\mathbf{C}(\dot{\mathbf{X}}, \mathbf{X})=\left[\begin{array}{cc}
\mathbf{0}_{3 \times 3} & \mathbf{0}_{3 \times 3} \\
\mathbf{0}_{3 \times 3} & \mathbf{S}^{T} \mathbf{I}_{\mathbf{g}} \dot{\mathbf{S}}+\mathbf{S}^{T}(\mathbf{S} \dot{\boldsymbol{\phi}})_{[X]}\left(\mathbf{I}_{\mathbf{g}} \mathbf{S}\right)
\end{array}\right]
$$

where $(\mathbf{S} \dot{\boldsymbol{\phi}})_{[X]}=(\boldsymbol{\omega})_{[X]}$ is the skew-symmetric matrix associated to $\boldsymbol{\omega}=\left[\begin{array}{lll}\omega_{x} & \omega_{y} & \omega_{z}\end{array}\right]^{T}$ defined by:

$$
(\boldsymbol{\omega})_{[X]}=\left[\begin{array}{ccc}
0 & -w_{z} & w_{y} \\
w_{z} & 0 & -w_{x} \\
-w_{y} & w_{x} & 0
\end{array}\right]
$$

With the assumption of elastic massless cables, the forces applied by the cables to the end-effector can be computed using the distances $l_{1}$ between cable end points and the cable free lengths $\mathbf{l}_{\mathbf{2}}$ (the length of the cable when its tension is zero). The free lengths can be calculated using the winch angular positions $\boldsymbol{\theta}: \mathbf{l}_{\mathbf{2}}=r \boldsymbol{\theta}+\mathbf{l}_{\mathbf{0}}$, where $l_{0}$ is the cable free lengths for the pose $\mathbf{X}=\mathbf{0}$ and $r$ is a scalar representing the winch radius.

The diagonal matrix of cable axial stiffness $\mathbf{K}=\operatorname{diag}\left(k_{i}\right)$ links cable tensions $\boldsymbol{\tau}$ to cable lengths:

$$
\tau=\mathbf{K}\left(\mathbf{l}_{2}\right)\left[\mathbf{l}_{1}-\mathbf{l}_{2}\right]
$$

where $k_{i}$ is the $i^{\text {th }}$ cable stiffness which depends on its free length $l_{2_{i}}$ (see Eq. (1)). All cables are assumed to be tensed. Dedicated algorithms have been proposed in the literature to guarantee nonnegative cable tensions in the CDPR workspace, e.g. [6], [9].

Finally, from (13), the dynamic model of a CDPR with massless elastic cables becomes:

$$
\mathbf{M}(\mathbf{X}) \ddot{\mathbf{X}}+\mathbf{C}(\dot{\mathbf{X}}, \mathbf{X}) \dot{\mathbf{X}}+\mathbf{G}=\tilde{\mathbf{J}}^{T}(\mathbf{X}) \mathbf{K}\left(\mathbf{l}_{\mathbf{2}}\right)\left[\mathbf{l}_{\mathbf{2}}-\mathbf{l}_{\mathbf{1}}(\mathbf{X})\right]
$$

Note that $\mathbf{l}_{\mathbf{1}}$ depends only on $\mathbf{X}$ since it is obtained by the inverse kinematics (computing the distances $A_{i} B_{i}$ being given the endeffector pose $\mathbf{X}$ ). Furthermore, the dependence of $\tilde{\mathbf{J}}^{T}$ on $\mathbf{X}$ has been made explicit in this equation.
2) Actuators: We consider a simplified model of the winch actuators. Indeed, in the usual case of a high reduction gear ratio (e.g. 210 on the setup presented in Section V), inertial couplings between the end-effector and the actuator rotors can be neglected (see Eq. 13.9 page 293 in [25] for more details). Furthermore, all actuators are considered to be identical, which is usually the case for CDPRs.

Let us define the following parameters:

- $B$ the rotor inertia,

- $F_{v}$ the viscous friction coefficient on the rotor axis,

- $\mathbf{F}_{\mathbf{s}}$ the dry friction vector on the rotor axes,

- $\gamma$ the vector of torques generated by electromagnetic interactions between the stator and rotor,

- $r$ the radius of all winches,

- $\boldsymbol{\alpha}$ the vector of rotor angular positions.

The tension of the part of the cable wound on the pulley is assumed to be null. Hence, $\mathbf{l}_{2}$ can be expressed as:

$$
\mathbf{l}_{2}=r \boldsymbol{\alpha}
$$

Then, the resulting simplified actuator dynamic equations are given by:

$$
B \ddot{\mathbf{l}}_{2}+F_{v} \dot{\mathbf{l}}_{2}+r \mathbf{F}_{\mathbf{s}}=r \boldsymbol{\gamma}+r^{2} \mathbf{K}\left(\mathbf{l}_{2}\right)\left[\mathbf{l}_{1}(\mathbf{X})-\mathbf{l}_{2}\right]
$$

\section{CONTROL}

This section introduces the two levels of control loops that are proposed to drive the CDPR end-effector along a prescribed smooth Cartesian trajectory.

Classical computed torque does not require a low-level velocity or position loop at the actuator level. Instead, the torques computed by the feedback linearization are directly sent to high bandwidth current loops. Since the bandwidth of a current loop (equal to the bandwidth of torque for an electrical motor) is many orders of magnitude larger than the mechanical bandwidth of most robots, its dynamics can be neglected. Nevertheless, when using high reduction ratios, significant dry friction occurs. In this case, direct torque control would be highly inaccurate since dry friction can represent up to $30 \%$ of the actuator nominal torque yielding very poor performances of the computed torque approach. This explains why computed torque is best suited to direct drive actuation.

In this work, we consider standard high reduction ratio actuators controlled by an inner position loop at the drive level. This servo loop is usually sampled at a high rate and may be considered as almost continuous time. Besides, the usual assumption of a high stiffness spring modeling of the cables is not made in this paper. Especially when using long cables or cables made of polymer material, this assumption does not hold.

Here, we assume that the actuator position loop has high gains and so a high bandwidth. The gains are tuned in such a way that the equivalent damping coefficient of the second order closed loop transfer function is equal to $1 / \sqrt{2}$ (optimal tuning to minimize the $5 \%$ rise time). The bandwidth of this inner position loop is supposed to be higher than the highest oscillation frequency of the CDPR end-effector. This is a mandatory condition for the design of a control law that is supposed to cope with these frequencies (for direct velocity feedback see [31], for lead controller see [32]). This results in a system with a fast variable, the actuator position loop, and a slow variable, the end-effector Cartesian position, coupled with cables treated as linear springs with moderate stiffness. Such a system typically fits into the singular perturbation framework. The latter will be used to demonstrate the stability of the proposed control law. One main contribution of this paper, which could be extended to types of flexible link robots other than CDPRs, is to propose a cascade control scheme for feedback linearization. The inner loop is a high 


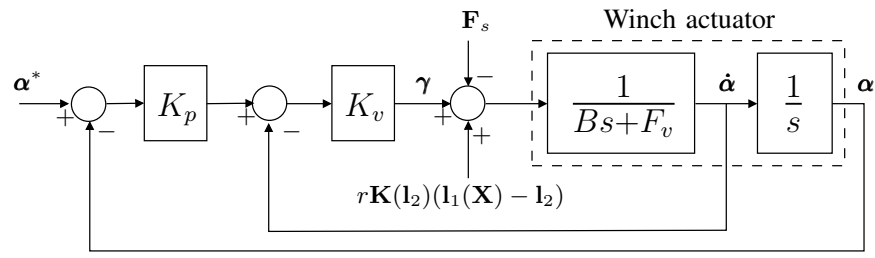

Fig. 2. Actuator position control loop.

gain actuator position loop designed to reject nonlinearities like dry friction. The outer loop achieves the feedback linearization based on the inverse dynamic model of the CDPR. The stability of the whole control scheme (Fig. 3) is proved using the singular perturbation theory in Section V.

\section{A. Actuator position loop}

All actuators are assumed to be controlled by a low-level position control loop, as shown in Fig. 2. Furthermore, an even lower level motor current loop allows for controlling $\gamma$ with a high bandwidth.

The control loop in Fig. 2 is usually carried out by a drive where the gains can be tuned through a dedicated software. The inner loop controls the rotor velocity and the outer loop controls the position.

Injecting the control law defined in Fig. 2 into the rotor dynamic model (17) gives:

$B \ddot{\mathbf{l}}_{2}+F_{v} \mathbf{i}_{2}=K_{v}\left(K_{p}\left(\mathbf{l}_{2}^{*}-\mathbf{l}_{2}\right)-\mathbf{i}_{2}\right)+r^{2} \mathbf{K}\left(\mathbf{l}_{2}\right)\left[\mathbf{l}_{1}(\mathbf{X})-\mathbf{l}_{2}\right]-r \mathbf{F}_{\mathbf{s}}$

where $\mathbf{l}_{2}^{*}=r \boldsymbol{\alpha}^{*}$.

By tuning the gains $K_{p}$ and $K_{v}$, it is possible to shape the position loop response to a step on $\mathbf{l}_{2}^{*}$.

\section{B. Feedback Linearization}

From (15), let us define the following feedback linearization control law for $\mathbf{l}_{2}^{*}$ :

$$
\begin{array}{r}
\tilde{\mathbf{J}}^{T}(\mathbf{X}) \mathbf{K}\left(\mathbf{l}_{\mathbf{2}}^{*}\right)\left[\mathbf{l}_{\mathbf{2}}^{*}-\mathbf{l}_{\mathbf{1}}(\mathbf{X})\right]=\mathbf{M}(\mathbf{X})\left[\ddot{\mathbf{X}}^{*}(t)\right. \\
\left.+\mathbf{K}_{v x}\left(\dot{\mathbf{X}}^{*}(t)-\dot{\mathbf{X}}\right)+\mathbf{K}_{p x}\left(\mathbf{X}^{*}(t)-\mathbf{X}\right)\right]+\mathbf{C}(\dot{\mathbf{X}}, \mathbf{X}) \dot{\mathbf{X}}+\mathbf{G}
\end{array}
$$

where $\mathbf{X}^{*}(t)$ is the desired Cartesian trajectory, $\mathbf{K}_{v x}$ and $\mathbf{K}_{p x}$ are tunable diagonal gain matrices. In the following equations, the dependency on time of the reference trajectory $\mathbf{X}^{*}(t)$ will be dropped for simplicity.

Using (1) and (14), the control law can be written:

$$
k_{s} \tilde{\mathbf{J}}^{T}(\mathbf{X})\left[\left[\begin{array}{c}
1 \\
\vdots \\
1
\end{array}\right]-\operatorname{diag}\left(\mathbf{l}_{\mathbf{2}}^{*}\right)^{-1} \mathbf{l}_{\mathbf{1}}(\mathbf{X})\right]=\mathbf{f}
$$

where

$$
\begin{aligned}
\mathbf{f}=\mathbf{M}(\mathbf{X})\left[\ddot{\mathbf{X}}^{*}+\mathbf{K}_{v x}\left(\dot{\mathbf{X}}^{*}-\dot{\mathbf{X}}\right)\right. & \left.+\mathbf{K}_{p x}\left(\mathbf{X}^{*}-\mathbf{X}\right)\right] \\
& +\mathbf{C}(\dot{\mathbf{X}}, \mathbf{X}) \dot{\mathbf{X}}+\mathbf{G}
\end{aligned}
$$

is the wrench to be applied to the end-effector to achieve the feedback linearization, and $\operatorname{diag}\left(\mathbf{l}_{2}^{*}\right)$ is the diagonal matrix constructed using the components of vector $\mathbf{l}_{2}^{*}$. In the most general case of a redundant CDPR (with more cables than degrees of freedom), isolating the terms in $\mathrm{l}_{2}^{*}$ yields to the non-unique solution:

$$
\operatorname{diag}\left(\mathbf{l}_{2}^{*}\right)^{-1} \mathbf{l}_{1}(\mathbf{X})=\left[\begin{array}{c}
1 \\
\vdots \\
1
\end{array}\right]+\frac{1}{k_{s}} \underbrace{\left[\left(-\tilde{\mathbf{J}}^{T}(\mathbf{X})\right)^{+} \mathbf{f}+\boldsymbol{\tau}_{\text {Null }}\right]}_{\boldsymbol{\tau}}
$$

where $\left(\tilde{J}^{T}\right)^{+}$is the Moore-Penrose pseudoinverse of the wrench matrix and $\boldsymbol{\tau}_{\text {Null }} \in \operatorname{Null}\left(\tilde{J}^{T}\right)$ is a tension vector. The vector $\left(-\tilde{\mathbf{J}}^{T}(\mathbf{X})\right)^{+} \mathbf{f}$ is the minimum-norm tension solution for (20), while the vector $\boldsymbol{\tau}_{\text {Null }}$ verifies $\tilde{\mathbf{J}}^{T} \boldsymbol{\tau}_{\text {Null }}=\mathbf{0}$. Through various dedicated algorithms [6], [9], the vector $\boldsymbol{\tau}_{\text {Null }}$ can be selected in the null space of the wrench matrix, such as to guarantee non-negative cable tensions $\tau \geq 0$. Note that this non-negative cable tension requirement enforces the requirement $\mathbf{l}_{2}^{*} \leq \mathbf{l}_{1}(\mathrm{X})$.

Finally, the analytic solution $\mathbf{l}_{2}^{*}\left(\mathbf{X}, \mathbf{X}^{*}\right)$ of the feedback linearization control signal is given by:

$$
\mathbf{l}_{2}^{*}=\left[\mathbf{I}+\frac{1}{k_{s}} \operatorname{diag}\left(\left(-\tilde{\mathbf{J}}^{T}(\mathbf{X})\right)^{+} \mathbf{f}+\boldsymbol{\tau}_{\text {Null }}\right)\right]^{-1} \mathbf{l}_{1}(\mathbf{X})
$$

Note that this equation relies on the measurements of $\mathbf{X}$ and $\dot{\mathbf{X}}$. In this work, these measurements are obtained from an external camera looking at the end-effector (eye-to-hand configuration [33]). A camera is used to estimate the Cartesian position $\mathbf{X}$ by tracking a marker attached to the CDPR end-effector. The 6-dimensional Cartesian velocity $\dot{\mathbf{X}}$ is estimated from the measurements of an Inertial Measurement Unit (IMU) embedded in the end-effector (Fig. 3).

\section{STABILITY ANALYSIS}

\section{A. Standard singular perturbation model}

The position controlled actuator dynamics and the feedback linearized end-effector dynamics can be written using the singular perturbation formalism [22]. The fast variable $\mathbf{z}$ is defined as follows:

$$
\mathbf{z}=\left[\begin{array}{l}
\mathbf{z}_{1} \\
\mathbf{z}_{2}
\end{array}\right]=\left[\begin{array}{c}
\left(\mathbf{l}_{2}^{*}-\mathbf{l}_{2}\right) / \epsilon \\
\mathbf{i}_{2}
\end{array}\right]
$$

with the small variable $\epsilon$ defined as $\epsilon=1 / K_{p}$ where $K_{p}$ is the high gain of the actuator position loop.

The slow variable $\mathbf{x}$ is the vector of the Cartesian position $\mathbf{X}$ of the end-effector and its time derivative $\dot{\mathbf{X}}$ :

$$
\mathbf{x}=\left[\begin{array}{l}
\mathbf{x}_{1} \\
\mathbf{x}_{2}
\end{array}\right]=\left[\begin{array}{l}
\mathbf{X} \\
\dot{\mathbf{X}}
\end{array}\right]
$$

Let us link gains $K_{p}$ and $K_{v}$ with the following equation:

$$
K_{v}=2 B K_{p}
$$

Thereby, it can be easily shown that the damping factor of the second order transfer function of the actuator position loop tends toward $1 / \sqrt{2}$ when $K_{p}$ goes to infinity. This pretty common tuning minimizes the $5 \%$ settling time of the step response.

Let the standard singular perturbation form be:

$$
\begin{aligned}
\dot{\mathbf{x}} & =f(t, \mathbf{x}, \mathbf{z}, \epsilon) \\
\epsilon \dot{\mathbf{z}} & =g(t, \mathbf{x}, \mathbf{z}, \epsilon)
\end{aligned}
$$

Thus, using (15), (18) and (26), the model can be put into the following standard singular perturbation form:

$$
\begin{aligned}
\dot{\mathbf{x}}= & {\left[\begin{array}{c}
\mathbf{x}_{2} \\
\mathbf{M}^{-1}\left(\mathbf{x}_{1}\right)\left[\tilde { \mathbf { J } } ^ { T } ( \mathbf { x } _ { 1 } ) \mathbf { K } ( \mathbf { l } _ { 2 } ^ { * } - \epsilon \mathbf { z } _ { 1 } ) \left[\mathbf{l}_{2}^{*}-\epsilon \mathbf{z}_{1}\right.\right. \\
\left.\left.-\mathbf{l}_{1}\left(\mathbf{x}_{1}\right)\right]-\mathbf{C}\left(\mathbf{x}_{1}, \mathbf{x}_{2}\right) \mathbf{x}_{2}-\mathbf{G}\right]
\end{array}\right] } \\
\epsilon \dot{\mathbf{z}}= & {\left[\begin{array}{c}
\mathbf{i}_{2}^{*}-\mathbf{z}_{2} \\
2\left(\mathbf{z}_{1}-\mathbf{z}_{2}\right)-\epsilon B^{-1}\left[F_{v} \mathbf{z}_{2}\right. \\
\left.-r^{2} \mathbf{K}\left(\mathbf{l}_{2}^{*}-\epsilon \mathbf{z}_{1}\right)\left[\mathbf{l}_{1}\left(\mathbf{x}_{1}\right)-\mathbf{l}_{2}^{*}+\epsilon \mathbf{z}_{1}\right]+r \mathbf{F}_{\mathbf{s}}\right]
\end{array}\right] }
\end{aligned}
$$




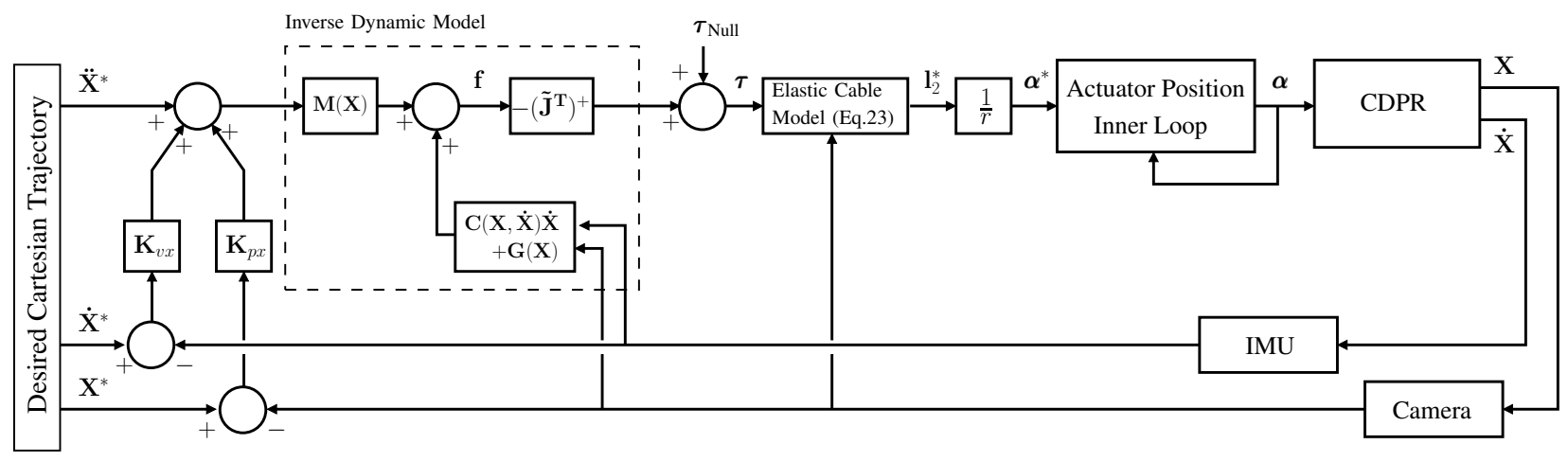

Fig. 3. Cartesian model-based control of elastic cable CDPRs, with an actuator position inner control loop.

Setting $\epsilon=0$ in (30) yields the fast transient of the variable $\mathbf{z}$ to converge to its unique root $h(t, \mathbf{x})=\left[\begin{array}{ll}\overline{\mathbf{z}}_{1}^{T} & \overline{\mathbf{z}}_{2}^{T}\end{array}\right]^{T}$ with $\overline{\mathbf{z}}_{1}=\overline{\mathbf{z}}_{2}=$ $\mathbf{i}_{2}^{*}\left(\mathbf{x}_{1}, \mathbf{X}^{*}\right)$ the quasi-steady state of the fast variable. Injecting these roots in (29), and setting $\epsilon=0$ as well, yields the slow subsystem or reduced model:

$$
\dot{\mathbf{x}}=\left[\begin{array}{c}
\mathbf{x}_{2} \\
\mathbf{M}^{-1}\left(\mathbf{x}_{1}\right)\left[\tilde{\mathbf{J}}^{T}\left(\mathbf{x}_{1}\right) \mathbf{K}\left(\mathbf{l}_{2}^{*}\right)\left[\mathbf{l}_{2}^{*}-\mathbf{l}_{1}\left(\mathbf{x}_{1}\right)\right]\right. \\
\left.-\mathbf{C}\left(\mathbf{x}_{2}, \mathbf{x}_{1}\right) \mathbf{x}_{2}-\mathbf{G}\right]
\end{array}\right]
$$

with $\mathbf{l}_{2}^{*}\left(\mathbf{X}, \mathbf{X}^{*}\right)$ verifying (19). When compared to (15), the free length $\mathbf{l}_{2}$ has been replaced by the free length reference $\mathbf{l}_{2}^{*}\left(\mathrm{X}, \mathrm{X}^{*}\right)$, which means that the winch actuator position control loops have an infinite bandwidth compared to the second order dynamics of the end-effector Cartesian position. This model simplifies further when the feedback linearization law (19) is applied:

$$
\dot{\mathbf{x}}=\left[\ddot{\mathbf{X}}^{*}+\mathbf{K}_{v x}\left(\dot{\mathbf{X}}^{*}-\mathbf{x}_{2}\right)+\mathbf{K}_{p x}\left(\mathbf{X}^{*}-\mathbf{x}_{1}\right)\right]
$$

With the change of variable $\mathbf{e}=\mathbf{X}^{*}-\mathbf{X}$, the reduced model becomes:

$$
\left[\begin{array}{c}
\dot{\mathbf{e}} \\
\ddot{\mathbf{e}}
\end{array}\right]=\left[\begin{array}{cc}
0 & 1 \\
-\mathbf{K}_{p x} & -\mathbf{K}_{v x}
\end{array}\right]\left[\begin{array}{c}
\mathbf{e} \\
\dot{\mathbf{e}}
\end{array}\right]
$$

whose origin $\{\mathbf{e}=\mathbf{0} ; \dot{\mathbf{e}}=\mathbf{0}\}$ is an exponentially stable equilibrium point if $\mathbf{K}_{v x}>0$ and $\mathbf{K}_{p x}>0$.

To study the fast transient of the variable $\mathbf{z}$ and its effective convergence toward its quasi-steady state $\overline{\mathbf{z}}$, a "stretched" time variable $t_{\epsilon}=\left(t-t_{0}\right) / \epsilon$ is introduced [23]. In this new time scale and at an initial time $t_{0}$, the dynamics of $\mathbf{z}$ is called the fast subsystem or boundary layer model of (30) and is given by:

$$
\frac{d \mathbf{y}}{d t_{\epsilon}}=\left[\begin{array}{c}
-\mathbf{y}_{2} \\
2\left(\mathbf{y}_{1}-\mathbf{y}_{2}\right)
\end{array}\right]
$$

with $\mathbf{y}=\left[\begin{array}{ll}\mathbf{y}_{1}^{T} & \mathbf{y}_{2}^{T}\end{array}\right]^{T}, \mathbf{y}_{1}=\mathbf{z}_{1}-\overline{\mathbf{z}}_{1}$ and $\mathbf{y}_{2}=\mathbf{z}_{2}-\overline{\mathbf{z}}_{2}$ a change of variable which purpose is to shift the equilibrium point toward zero. The boundary layer model (34) is a linear system. The eigenvalues of the matrix $\mathbf{A}$ of its state-space representation are equal to $-1 \pm j$. Thus, the origin of the boundary layer model is exponentially stable with a damping factor equal to $\frac{1}{\sqrt{2}}$.

\section{B. Tikhonov's Theorem}

Theorem 11.2 in [22] states that the solution of (29) and (30) approaches the solution of the reduced model (32) if:

- the origin is an exponentially stable equilibrium point of the boundary-layer model uniformly in $(t, \mathbf{x})$;
- the origin is an exponentially stable equilibrium point of the reduced model;

- on any compact subset including the origin, the functions $f, g$, their first partial derivatives with respect to $(\mathbf{x}, \mathbf{z}, \epsilon)$, and the first partial derivative of $g$ with respect to $t$ are continuous and bounded, $h(t, \mathbf{x})$ and $[\partial g(t, \mathbf{x}, \mathbf{z}, 0) / \partial \mathbf{z}]$ have bounded first partial derivatives with respect to their arguments, and $[\partial f(t, \mathbf{x}, h(t, \mathbf{x}), 0) / \partial \mathbf{x}]$ is Lipschitz in $\mathbf{x}$, uniformly in $t$; the initial data are smooth functions of $\epsilon$.

The first two points were verified in the previous section. The conditions in the third point can be easily verified if the reference trajectory $\mathbf{X}^{*}$ is within the wrench-feasible workspace of the CDPR.

If all conditions are verified, the theorem states that, for gains $K_{v}$ and $K_{p}$ high enough ( $\epsilon$ small enough), for $t>t_{0}>0$, the singular perturbation problem (29)-(30) has a unique solution $\mathbf{x}(t, \epsilon)$ and $\mathbf{z}(t, \epsilon)$ such that:

$$
\begin{aligned}
\mathbf{x}(t, \epsilon)-\overline{\mathbf{x}}(t) & =\mathcal{O}(\epsilon) \\
\mathbf{z}(t, \epsilon)-h(t, \overline{\mathbf{x}}(t))-\hat{\mathbf{y}}(t / \epsilon) & =\mathcal{O}(\epsilon)
\end{aligned}
$$

with $\overline{\mathbf{x}}$ the solution of the reduced model (32) and $\hat{\mathbf{y}}$ the solution of the boundary layer (34) or, equivalently, using the variable e:

$$
\begin{aligned}
\mathbf{e}(t, \epsilon)-\overline{\mathbf{e}}(t) & =\mathcal{O}(\epsilon) \\
\mathbf{z}(t, \epsilon)-h(t, \overline{\mathbf{e}}(t))-\hat{\mathbf{y}}(t / \epsilon) & =\mathcal{O}(\epsilon)
\end{aligned}
$$

where $\overline{\mathbf{e}}$ is the solution of (33). Furthermore, a $t_{b}$ exists such that for $t>t_{b}>t_{0}$ :

$$
\begin{aligned}
\mathbf{e}(t, \epsilon) & =\overline{\mathbf{e}}(t)+\mathcal{O}(\epsilon) \\
\mathbf{z}(t, \epsilon) & =h(t, \overline{\mathbf{e}}(t))+\mathcal{O}(\epsilon) \\
& =\left[\begin{array}{c}
\mathbf{l}_{2}^{*}\left(\mathbf{X}, \mathbf{X}^{*}\right) \\
O
\end{array}\right]+\mathcal{O}(\epsilon)
\end{aligned}
$$

Consequently, the feedback linearized system controlled with lowlevel high gain position loops is stable at the origin since $\lim _{t \rightarrow \infty} \overline{\mathbf{e}}(t)=\mathbf{0}$ and $\mathbf{e}(t, \epsilon)$, the trajectory tracking error, is at an order of magnitude $\mathcal{O}(\epsilon)$ equal to $\overline{\mathbf{e}}(t)$.

\section{EXPERIMENTS ${ }^{1}$}

\section{A. The PiSaRo2 robot}

The PiSaRo2 is a 3-cable suspended planar CDPR inspired by [34] (see Fig. 4).

\footnotetext{
${ }^{1}$ This paper has supplementary downloadable material available at http://ieeexplore.ieee.org. It includes a video presenting the setup used to carry out the experiments and showing the main experimental results, a summary file and a readme file. The size of this material is $33.2 \mathrm{MB}$.
} 


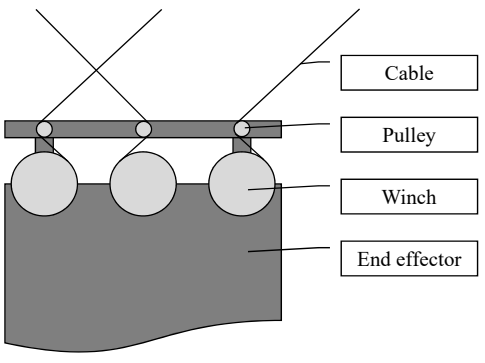

Fig. 4. PiSaRo2 cable configuration.

The PiSaRo2 structure is made of Matrix ${ }^{\mathrm{TM}}$ aluminum beams. This allows for rapid prototyping of its architecture while keeping a decent global rigidity. Its workspace has been optimized to cover a $1.2 \mathrm{~m}$ by $0.96 \mathrm{~m}$ white board.

Three winches are driven by three Dynamixel ${ }^{\mathrm{TM}} \mathrm{XM} 430-\mathrm{W} 210-\mathrm{R}$ actuators mounted on the end-effector. The inner loop of the proposed control strategy sends control inputs to the built-in velocity control loops of the Dynamixels ${ }^{\mathrm{TM}}$, which have been activated (the gain of this loop is set to 180 at the Dynamixel ${ }^{\mathrm{TM}}$ register address 78 ).

The position loop is tuned to maximize the bandwidth while keeping the step response overshoot around $5 \%$ yielding $K_{p}=125$. With this setting, the winch position loop exhibits a $-3 \mathrm{~dB}$ bandwidth of $21 \mathrm{~Hz}$. These actuators are connected to a Raspberry Pi model 3 (RPi) by a $1 \mathrm{Mbps}$ RS485 serial bus. The RPi is a target for a dedicated toolbox developed in our lab called RPIt ${ }^{2}$. With RPIt, it is possible to use Matlab's ${ }^{\mathrm{TM}}$ hardware-in-the-loop Simulink Coder ${ }^{\mathrm{TM}}$ rapid prototyping toolbox with a RPi target. Generic blocks are available to control Dynamixel actuators, to read measurements from standard IMU chips connected to the RPi i2c bus or to interact through TCPIP sockets with a dedicated vision PC.

The winches were printed on a Stratasys 4 Polyjet $^{\mathrm{TM}}$ 3D printer using VeroClear or VeroWhite polymer. A helicoidal track at the bottom of the winch groove helps to guide the cable winding, avoiding sudden cable jump from one layer to another. Brakes have been added to prevent the robot from falling in case of power loss due to its own mass (see Fig. 5).

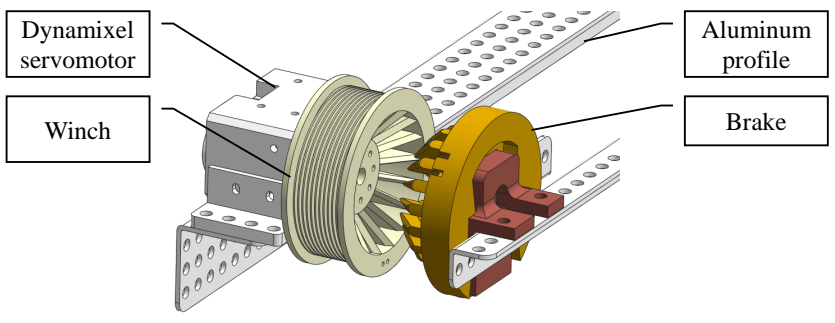

Fig. 5. PiSaRo2 winches.

The robot is suspended with three fishing wires (Caperlan ${ }^{\mathrm{TM}}$ Line Clear $0.55 \mathrm{~mm}, 13.8 \mathrm{~kg}$ ). Cyclic tension tests show that after a running-in procedure, its specific stiffness $k_{s}$ tends toward $500 \mathrm{~N}$.

A picture of the end-effector is given in Fig. 6. Four red LED markers arranged in a square pattern are used to simplify the pose estimation algorithm on the vision PC. An Invensense ${ }^{\mathrm{TM}}$ MPU 9150 IMU is positioned approximately at the end-effector center of gravity. A USB wireless Xbox $360^{\mathrm{TM}}$ controller receiver is used for manual remote control of the robot.

${ }^{2}$ https://github.com/jacqu/rpit

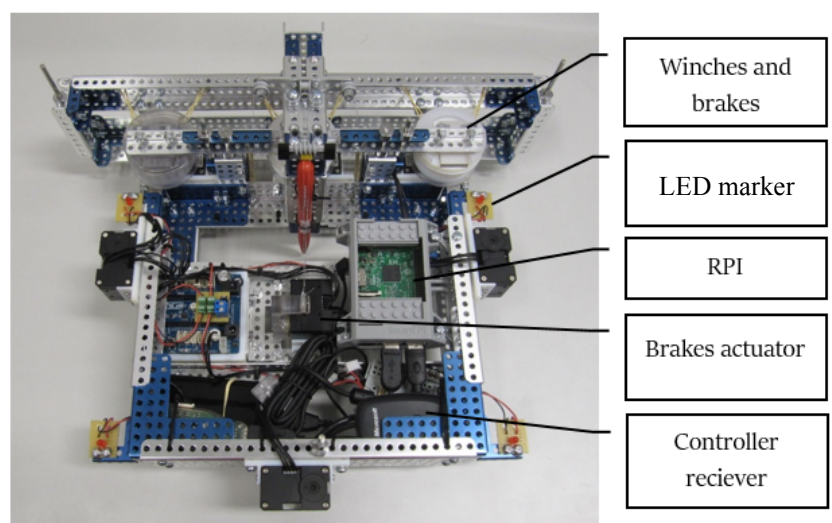

Fig. 6. PiSaRo2 end-effector.

This robot weights $3.2 \mathrm{~kg}$, can theoretically carry a load of $2 \mathrm{~kg}$ within a $1.2 \mathrm{~m} \times 1 \mathrm{~m}$ workspace, has a maximum speed of approximately $0.25 \mathrm{~m} \mathrm{~s}^{-1}$ and a maximum position accuracy of $0.1 \mathrm{~mm}$ (related to encoder resolution).

\section{B. $500 \mathrm{~Hz}$ visual feedback}

A vision $\mathrm{PC}$ is dedicated to the image processing at a high sampling rate $(500 \mathrm{~Hz})$. It is connected through USB3 to a Ximea $\mathrm{xiQ}^{\mathrm{TM}}$ MQ003MG-CM VGA monochrome 500 frames per second camera mounted on a tripod and pointed to the CDPR end-effector. It acts as a server for a special RPIt block that communicates using TCPIP sockets. To keep the system fully wireless while minimizing jitters and delays, a dedicated $5 \mathrm{GHz}$ MIMO WiFi hotspot is used to relay the packets from the RPi to the vision PC. Delays were assessed and long lasting high rate ping sessions show that they are less than $1 \mathrm{~ms}$.

The visual control loop runs on the RPi with a sampling rate of $100 \mathrm{~Hz}$. It periodically sends measurement requests through a socket to the vision PC. Since the visual measurement runs five times faster than the visual control loop and since it is not synchronized with the camera clock, there is a random delay in the visual feedback loop ranging from 0 to $2 \mathrm{~ms}$ with an average of $1 \mathrm{~ms}$. We assume that this delay is negligible with respect to the control loop sampling period (10 ms).

The image processing is a simple OpenCV blob detector that robustly detects 4 LED spots in the image. With the pose estimation, it takes less than $400 \mu$ s on the vision PC (Intel Core ${ }^{\mathrm{TM}}$ i5-6500 CPU @ $3.20 \mathrm{GHz}$ ). Video stream buffering delay of the Ximea Linux device driver has also been assessed and can be considered as negligible.

\section{Experiments}

The feedback linearization of (23) is implemented. The reference trajectory $\mathbf{X}^{*}$ describes a $21 \mathrm{~cm} \times 21 \mathrm{~cm}$ square on the white board. Though twice differentiable with respect to time, $\mathbf{X}^{*}$ has high accelerations around the square corner in order to excite the flexible modes of the robot. Dynamic Visual Servoing (DVS) implementing the control law in (23) is compared with two other control schemes: Inverse Kinematics Control (IKC) and Kinematic Visual Servoing (KVS).

IKC calculates offline the inverse kinetostatic model of the desired Cartesian trajectory $\mathbf{X}^{*}$. It provides an equivalent quasi-static trajectory at the actuator level which is replayed with proprioceptive (encoder) feedback but without exteroceptive (vision) feedback.

KVS assumes that the robot is a perfect Cartesian motion device, i.e., dynamic effects are neglected (see [3]). A position-based visual servo loop is implemented that uses the Jacobian $\tilde{\mathbf{J}}$ as the interaction 
matrix (see (9)). The visual feedback provides the measurement of $\mathbf{X}$ with respect to 3 degrees of freedom: the two translations within the robot plane and the rotation around an axis normal to this plane.

First experiments of DVS with the control law (23) exhibit significant tracking errors (up to $2.5 \mathrm{~cm}$ ). As the feedback linearized outer loop is equivalent to a double integrator system, adding an integral gain in the proportional derivative controller should be worthless. However, an integral effect improved tracking error by compensating for small unmodeled phenomena in this control strategy [1]. Consequently, to cope with uncertainties on the cable stiffness amongst others, the control law (21) has been modified accordingly with:

$$
\begin{array}{r}
\mathbf{f}=\mathbf{M}(\mathbf{X})\left[\ddot{\mathbf{X}}^{*}+\mathbf{K}_{v x}\left(\dot{\mathbf{X}}^{*}-\dot{\mathbf{X}}\right)+\mathbf{K}_{p x}\left(\mathbf{X}^{*}-\mathbf{X}\right)\right. \\
\left.+\mathbf{T}_{i} \int\left(\mathbf{X}^{*}-\mathbf{X}\right) d t\right]+\mathbf{C}(\dot{\mathbf{X}}, \mathbf{X}) \dot{\mathbf{X}}+\mathbf{G}
\end{array}
$$

where $\mathbf{T}_{i}$ is the diagonal matrix of the integral term gains. In order to prove the stability of the whole system, the stability of the reduced model (31) with the modified PID feedback linearization (42) should be proved. This can be easily done since the resulting system is linear. In our experiments the PID gains have been tuned as follows:

$$
\begin{aligned}
\mathbf{K}_{p x} & =\operatorname{diag}(144,144,80) \\
\mathbf{K}_{v x} & =\operatorname{diag}(10,10,5) \\
\mathbf{T}_{i} & =\operatorname{diag}(300,300,300)
\end{aligned}
$$

These gains where tuned experimentally to minimize the tracking error along the trajectory (a $21 \mathrm{~cm} \times 21 \mathrm{~cm}$ square described at an average speed of $0.11 \mathrm{~m} \mathrm{~s}^{-1}$ ), while avoiding actuator saturation. These gains yield a $-3 \mathrm{~dB}$ bandwidth of $0.41 \mathrm{~Hz}$ for the two translational control loops and $1.75 \mathrm{~Hz}$ for the rotational control loop. Note that the $-3 \mathrm{~dB}$ bandwidth of the winch angular position loop $(21 \mathrm{~Hz})$ is much larger. This verifies the main assumption of a twotime-scale system used by the singular perturbation theory to achieve the stability proof.

Estimates of the Cartesian position $\mathbf{X}$ and velocity $\dot{\mathbf{X}}$ are required to compute (42). The Cartesian position is measured by the vision system whereas the velocity is estimated from the measurements of an IMU. One axis of the IMU gyro gives the velocity directly around the axis normal to the robot plane. The translational velocities are obtained after integration over time of the accelerometer axes which are in the robot plane. In order to avoid a drift of this estimate over time, it is processed by a high-pass digital filter.

Fig. 7, 8 and 9 show the $2 \mathrm{D}$ end-effector trajectory and the quadratic tracking error for a square-shaped $\mathbf{X}^{*}(t)$ that is followed at an average speed of $0.11 \mathrm{~m} \mathrm{~s}^{-1}$. These plots show clearly the superiority of the DVS control law. KVS has increased significant errors over time due to the absence of oscillation rejection (the robot dynamics are neglected). IKC has greater tracking error than DVS as well due to its lack of exteroceptive feedback. Indeed, modeling errors that impact the inverse kinetostatic model cannot be rejected with this open-loop approach. On the other hand, it yields less oscillations than KVS since its stability is not compromised by a feedback loop designed for a simplified model.

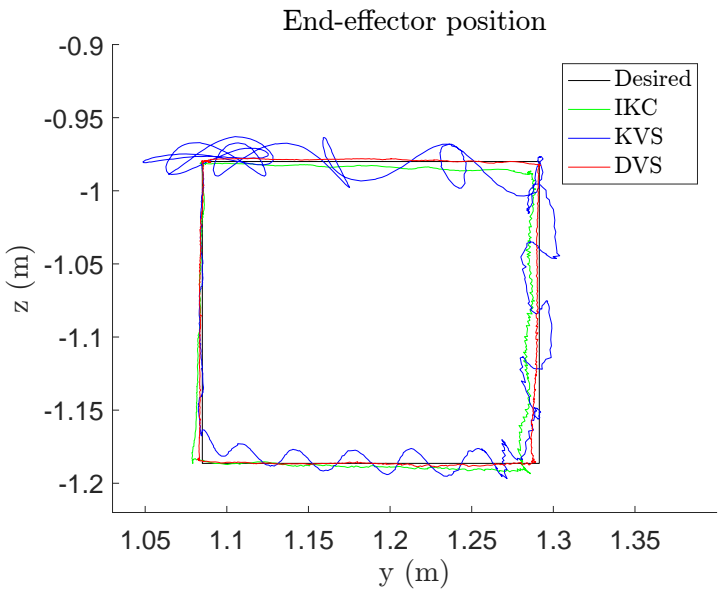

Fig. 7. End-effector trajectory with IKC (green), KVS (blue) and DVS (red).

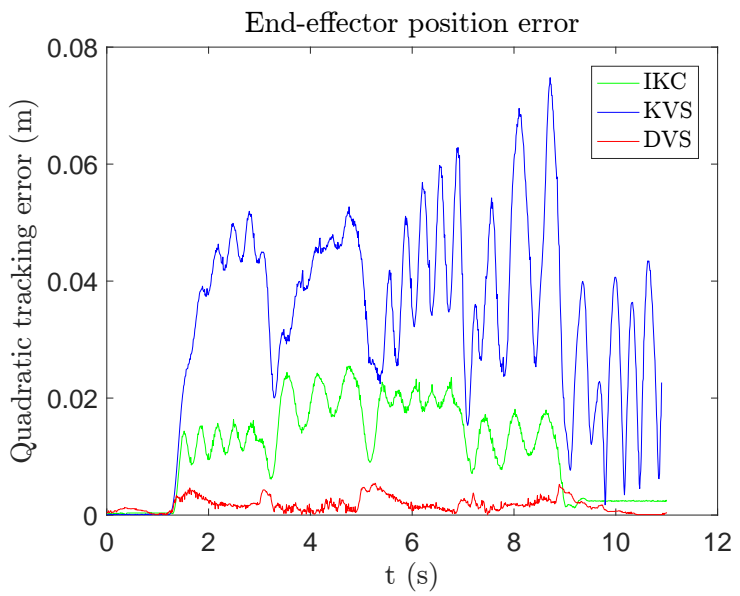

Fig. 8. Quadratic tracking error: IKC (green), KVS (blue) and DVS (red).

The RMS errors of the end-effector position along the whole trajectory are respectively $32.6 \mathrm{~mm}, 11.2 \mathrm{~mm}$ and $1.7 \mathrm{~mm}$ with KVS, IKC and DVS.

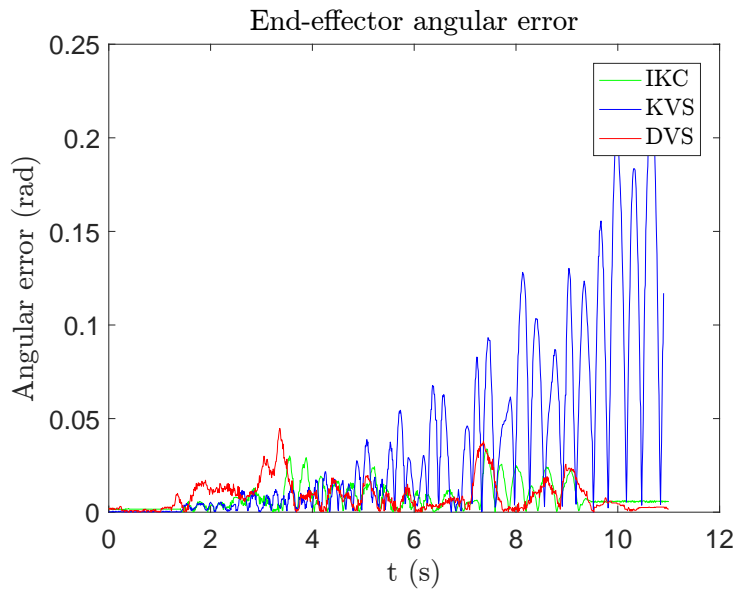

Fig. 9. Absolute value of the angular error: IKC (green), KVS (blue) and DVS (red).

The mean anglular errors along the whole trajectory are respectively $36.3 \mathrm{mrad}, 8.6 \mathrm{mrad}$ and $7.8 \mathrm{mrad}$ with KVS, DVS and IKC. 


\section{CONCLUSION}

In this paper, a new approach to dynamic control of CDPRs using visual feedback has been proposed. CDPRs are usually controlled using a torque control input through the actuator drive current loop, thereby acting on the cable force. This approach is relevant for robots with high stiffness cables. In this case, the proof of stability using the singular perturbation theory is almost the same as for other kinds of flexible robots, parallel [35] or serial [21], [36], and the small variable is usually chosen inversely proportional to the stiffness coefficient. However, when using long cables or low stiffness cables, like cables made of polymers, this assumption does not hold anymore. Thus, as it is usually the case with industrial robots, we propose to control the CDPR in a cascaded way, with a linearizing inner loop controlling the winch angular position and an outer visual loop controlling the end-effector Cartesian position. A new singular perturbation small variable is introduced i.e. the inverse of the position loop gain. Using Tikhonov's theorem, the stability of this dynamic visual servoing control scheme implementing standard feedback linearization was demonstrated in this paper without requiring a high-stiffness cable assumption. Experiments validated the approach on a planar 3cable CDPR, showing the added value of a dynamic control law with respect to a kinematic one when using high frame rate visual feedback.

\section{ACKNOWLEDGMENT}

This work is part of project DexterWide (ANR-15-CE10-0006) funded by the French National Research Agency (ANR).

\section{REFERENCES}

[1] F. Paccot, N. Andreff, and P. Martinet, "A review on the dynamic control of parallel kinematic machines: Theory and experiments," The International Journal of Robotics Research, vol. 28, no. 3, pp. 395-416, 2009.

[2] L. Weiss, A. Sanderson, and C. Neuman, "Dynamic sensor-based control of robots with visual feedback," IEEE Journal on Robotics and Automation, vol. 3, no. 5, pp. 404-417, October 1987.

[3] F. Chaumette and S. Hutchinson, "Visual servo control. I. basic approaches," IEEE Robotics Automation Magazine, vol. 13, no. 4, pp. 82 90, Dec 2006.

[4] B. Espiau, F. Chaumette, and P. Rives, "A new approach to visual servoing in robotics," IEEE Transactions on Robotics and Automation, vol. 8, no. 3, pp. 313-326, Jun 1992.

[5] H. Cheng, Y.-K. Yiu, and Z. Li, "Dynamics and control of redundantly actuated parallel manipulators," IEEE/ASME Transactions on Mechatronics, vol. 8, no. 4, pp. 483-491, Dec 2003.

[6] S.-R. Oh and S. Agrawal, "Cable suspended planar robots with redundant cables: controllers with positive tensions," IEEE Transactions on Robotics, vol. 21, no. 3, pp. 457-465, Jun. 2005.

[7] P. H. Borgstrom, B. L. Jordan, G. S. Sukhatme, M. A. Batalin, and W. J. Kaiser, "Rapid computation of optimally safe tension distributions for parallel cable-driven robots," IEEE Transactions on Robotics, vol. 25, no. 6, pp. 1271-1281, 2009.

[8] H. D. Taghirad and Y. B. Bedoustani, "An analytic-iterative redundancy resolution scheme for cable-driven redundant parallel manipulators," IEEE Transactions on Robotics, vol. 27, no. 6, pp. 1137-1143, 2011.

[9] M. Gouttefarde, J. Lamaury, C. Reichert, and T. Bruckmann, "A versatile tension distribution algorithm for $n$-dof parallel robots driven by $n+2$ cables," IEEE Transactions on Robotics, vol. 31, no. 6, pp. 1444-1457, Dec 2015.

[10] S. Kawamura, H. Kino, and C. Won, "High-speed manipulation by using parallel wire-driven robots," Robotica, vol. 18, no. 1, pp. 13-21, 2000.

[11] S. Fang, D. Franitza, M. Torlo, F. Bekes, and M. Hiller, "Motion control of a tendon-based parallel manipulator using optimal tension distribution," IEEE/ASME Transactions on Mechatronics, vol. 9, no. 3, pp. 561-568, Sept 2004.

[12] R. L. Williams II and P. Gallina, "Translational planar cable-direct-driven robots," Journal of Intelligent and Robotic Systems, vol. 37, no. 1, pp. 69-96, May 2003.
[13] H. Kino, T. Yahiro, F. Takemura, and T. Morizono, "Robust PD control using adaptive compensation for completely restrained parallel-wire driven robots: Translational systems using the minimum number of wires under zero-gravity condition," IEEE Transactions on Robotics, vol. 23, no. 4, pp. 803-812, Aug 2007.

[14] R. Babaghasabha, M. A. Khosravi, and H. D. Taghirad, "Adaptive robust control of fully-constrained cable driven parallel robots," Mechatronics, vol. 25 , pp. $27-36,2015$.

[15] X. Diao and O. Ma, "Vibration analysis of cable-driven parallel manipulators," Multibody System Dynamics, vol. 21, no. 4, pp. 347-360, May 2009.

[16] G. Meunier, B. Boulet, and M. Nahon, "Control of an overactuated cable-driven parallel mechanism for a radio telescope application," IEEE Transactions on Control Systems Technology, vol. 17, no. 5, pp. 10431054, Sept 2009.

[17] R. J. Caverly and J. R. Forbes, "Dynamic modeling and noncollocated control of a flexible planar cable-driven manipulator," IEEE Transactions on Robotics, vol. 30, no. 6, pp. 1386-1397, Dec 2014.

[18] _ - "Flexible cable-driven parallel manipulator control: Maintaining positive cable tensions," IEEE Transactions on Control Systems Technology, vol. PP, no. 99, pp. 1-10, 2017.

[19] M. A. Khosravi and H. D. Taghirad, "Dynamic modeling and control of parallel robots with elastic cables: Singular perturbation approach," IEEE Transactions on Robotics, vol. 30, no. 3, pp. 694-704, June 2014.

[20] H. Jamshidifar, S. Khosravani, B. Fidan, and A. Khajepour, "Vibration decoupled modeling and robust control of redundant cable-driven parallel robots," IEEE/ASME Transactions on Mechatronics, vol. 23, no. 2, pp. 690-701, April 2018.

[21] F. Ghorbel, J. Y. Hung, and M. W. Spong, "Adaptive control of flexiblejoint manipulators," IEEE Control Systems Magazine, vol. 9, no. 7, pp. 9-13, Dec 1989.

[22] H. K. Khalil, Nonlinear Systems, 3rd ed. Pearson, Dec. 2001, 750 pages.

[23] P. Kokotovic, H. Khalil, and J. O'Reilly, Singular Perturbation Methods in Control: Analysis and Design. Society for Industrial and Applied Mathematics, 1999.

[24] X. Weber, L. Cuvillon, and J. Gangloff, "Active vibration canceling of a cable-driven parallel robot in modal space," in 2015 IEEE International Conference on Robotics and Automation, May 2015, pp. 1599-1604.

[25] B. Siciliano and O. Khatib, Springer Handbook of Robotics. Springer, 2008.

[26] H. Vallery, J. Veneman, E. van Asseldonk, R. Ekkelenkamp, M. Buss, and H. van Der Kooij, "Compliant actuation of rehabilitation robots," IEEE Robotics Automation Magazine, vol. 15, no. 3, pp. 60-69, September 2008.

[27] P. Agarwal, Y. Yun, J. Fox, K. Madden, and A. D. Deshpande, "Design, control, and testing of a thumb exoskeleton with series elastic actuation," The International Journal of Robotics Research, vol. 36, no. 3, pp. 355375,2017

[28] A. Calanca, R. Muradore, and P. Fiorini, "A review of algorithms for compliant control of stiff and fixed-compliance robots," IEEE/ASME Transactions on Mechatronics, vol. 21, no. 2, pp. 613-624, April 2016.

[29] N. Riehl, M. Gouttefarde, C. Baradat, and F. Pierrot, "On the determination of cable characteristics for large dimension cable-driven parallel mechanisms," in 2010 IEEE International Conference on Robotics and Automation, 2010, pp. 4709-4714.

[30] N. Riehl, "Modélisation et design de robots parallèles à câbles de grande dimension,” Ph.D. dissertation, Université Montpellier 2, May 2011.

[31] C. J. Goh and T. K. Caughey, "On the stability problem caused by finite actuator dynamics in the collocated control of large space structures," International Journal of Control, vol. 41, no. 3, pp. 787-802, 1985.

[32] A. Preumont, "Vibration Control of Active Structures," in Vibration Control of Active Structures, ser. Solid Mechanics and Its Applications. Dordrecht: Springer Netherlands, 2011, vol. 179.

[33] F. Chaumette and S. Hutchinson, "Visual servo control, part II: Advanced approaches," IEEE Robotics and Automation Magazine, vol. 14, no. 1, pp. 109-118, 2007.

[34] X. Jiang and C. Gosselin, "Trajectory generation for three-degree-offreedom cable-suspended parallel robots based on analytical integration of the dynamic equations," Journal of Mechanisms and Robotics, vol. 8, no. 4, p. 041001, 2016.

[35] T. Sun, D. Liang, and Y. Song, "Singular-perturbation-based nonlinear hybrid control of redundant parallel robot," IEEE Transactions on Industrial Electronics, vol. 65, no. 4, pp. 3326-3336, April 2018.

[36] B. Siciliano and W. J. Book, "A singular perturbation approach to control of lightweight flexible manipulators," The International Journal of Robotics Research, vol. 7, no. 4, pp. 79-90, 1988. 